\title{
Focus Point on Modern Astronomy: Selected Issues in Nuclear and High Energy Astrophysics
}

Published online: 19 November 2018 - C Società Italiana di Fisica / Springer-Verlag GmbH Germany, part of Springer Nature, 2018

Modern Astronomy is a multidisciplinary science that evolved widely with respect to the old traditional and romantic discipline made at a telescope, observing stars and taking notes of their movements in the sky. Nowadays, high-resolution stellar spectra from gigantic reflectors like VLT, images of planets and distant galaxies made at infrared wavelengths where cool matter or redshifted objects are best seen, high-definition maps of galaxies and the cosmos provided by space-borne telescopes are invaluable sources of data. However, they give us only a partial vision of the universe, which, to be studied and understood, needs to be scrutinised not only in the electromagnetic spectrum but also through probes of different nature, such as high energy particles (cosmic rays) accelerated by galactic mechanisms, neutrinos from nuclear processes, and gravitational waves from space-time perturbations. In this much broader picture, "classical" astronomers, stellar physicists, experts of nucleosynthesis, nuclear and particle physicists and geochemists work together to study the universe and understand its formation and evolution.

Since many experts in different fields are needed to undertake this arduous task, it is crucial that the training of young researchers be focused both on providing them with a general physical background, and on specialising them in some specific field among those mentioned. This book aims to give the readers an overview on the state of the art of research in some crucial fields of astrophysics, in the epoch in which detection of individual neutrinos and of gravitational waves born from compact stellar objects are founding the multi-messenger era of cosmic studies. In order do this, the present volume collects a number of invited contributions on the highly debated subjects in stellar modelling and nucleosynthesis, in $\gamma$ - and X-ray astronomy, in astro-particle physics, and in experimental low-energy nuclear astrophysics. 